\title{
Typhlitis, CTCAE 5.0
}

National Cancer Institute

\section{Source}

National Cancer Institute. Iyphlitis, CT CAE 5.0. NCI Thesaurus. Code C146730.

A disorder characterized by necrotizing enterocolitis in neutropenic patients. 